\title{
Geo-localization System for People with Cognitive Disabilities
}

\author{
João Ramos, Ricardo Anacleto, Paulo Novais, Lino Figueiredo, Ana Almeida and \\ José Neves
}

\begin{abstract}
Technology is present in almost every simple aspect of the people's daily life. As an instance, let us refer to the smartphone. This device is usually equipped with a GPS module which may be used as an orientation system, if it carries the right functionalities. The problem is that these applications may be complex to operate and may not be within the bounds of everybody.

Therefore, the main goal here is to develop an orientation system that may help people with cognitive disabilities in their day-to-day journeys, when the caregivers are absent. On the other hand, to keep paid helpers aware of the current location of the disable people, it will be also considered a localization system. Knowing their current locations, caregivers may engage in others activities without neglecting their prime work, and, at the same time, turning people with cognitive disabilities more independent.
\end{abstract}

Key words: Cognitive Disabilities, Mobile Communication, Localization, Orientation, Persons Tracking, Ambient Intelligence

\section{Introduction}

Increasing people, elder and sick, is becoming a real problem to the social security systems of developed countries. Indeed, due to the rise of life expectancy and the reduced number of births, the population is getting older [1], therefore requiring more care, which means more costs to the health system's. To reduce the impact of

João Ramos, Paulo Novais, José Neves

Informatics Department, University of Minho, Portugal, e-mail: \\{jramos, pjon, jneves $\backslash$

\} @di. uminho.pt

Ricardo Anacleto, Lino Figueiredo, Ana Almeida

GECAD - Knowledge Engineering and Decision Support, ISEP, Portugal e-mail: \\{rmao, $1 \mathrm{~b} f$, amn \\} @isep.ipp.pt 
this situation there are a few protection services and facilities, like nursing homes or caregivers. This type of services involves a significant loss of mobility by the patient. This means that the elder leaves his/her house and/or that a person (that may be a family member) moves in.

To prevent or minimize this independence loss there are smart houses [2], which are considered a good alternative, since they use embedded devices that control the patient health and enable remote access to such data by the physician or other caregiver $[3,4,5]$. But outside his/her home this technology isn't available and the patient can not be monitored.

In the medical arena there have been a number of absorbing developments; indeed some diagnostic techniques have been enhanced and others have been created/discovered. Thereby the well-being of individuals and societies suffered a change, for the better. However, there are some diseases that still do not have a cure, such as the ones known as cognitive disabilities. To this type of people it is very complicated to go out alone, to whom orientation may become a very difficult task and help is needed.

Since 1988 assisted technologies have gain attentiveness. As expected, field experts turned their attention on how it may improve the people's quality of life with cognitive impairments [6]. Nevertheless, a set of devices have been developed, but the problem with such tools is that they were advanced to be embedded in the home environment (e.g., smart houses). Like an ordinary person, people with cognitive disabilities may leave their premises and, once outside, this technology may not able to be used or obtained.

However, there are researchers that have been developing new ways to use the available technology outside a premise, i.e., looking at new tools. Such devices need to be easy-to-use, small, lightweight and resistant, otherwise they may not last for a long time $[7,8]$.

Our development described in [9] not only enables the positioning for this type of people but also allows the caregiver to know the current location of persons with cognitive disabilities. Thus, the independence of people with cognitive disabilities is increased and the same occurs to their caregiver(s) that may have other work without neglecting the care provision.

In section 2 is presented work developed by other authors. Section 3 describes the proposed system, including all its features. Finally, at section 4 a brief reflection about this work is presented.

\section{Related Work}

A system that may help people with cognitive disabilities outside their premises was developed by Carmien et al. [10]. The authors, based on traditional orientation methods, developed a system that enables human beings to use the public transportation system. The person with cognitive disabilities with a smartphone may make use of the bus to travel from one particular place to another. The reduced number of routes 
that were drawn in a simple map is surpassed and the user may travel to a greater set of destinations.

This project had two main goals. The former had in mind to provide assistance to the user through just-in-time information about the travel path (including routes and the bus that should be reached for and hold). The extra goal aims to a simple and fast way of a process of communication between the user and a caregiver. The position of each bus is given in real-time by a GPS module previously installed on it. This position is then sent to a server. The route is calculated based on the information presented on the server.

Besides the previous described system the authors also developed a second prototype that did not need a support structure. Using an end-user programming tool the caregiver could create scripts according to the activities to be carried out by the person with cognitive disabilities.

In 2009, Liu et al. [11] developed an orientation system for handicapped people. On their study the players freely use the prototype, while being remotely controlled, i.e., the users could walk and explore all the system functionalities.

Liu et al. look at two different studies. The former tries to identify which features of the indoor system should be extrapolated to the outdoor one. Combining pictures with overlaid arrows, audio and text messages, the user could successfully attain a particular destination. Outside the premises, the orientation is more complicated since it is a more dynamic environment (e.g., traffic and the determination of the relative position of something or someone passing by), which may turn the system unreliable. The second study examines the usability of landmarks in orientation of people with cognitive disabilities. The obtained results show that there are a few considerations that must be taken into consideration when orientation is provided. For example, a near landmark should be used instead of a marker that is outside the user view (e.g., behind a tall building). This landmark should also be presented as a picture in the user's prototype to help him/her to identify it. If the image is hard to associate to the real landmark, the user may become stressed and confused and the orientation system may fail.

AlzNav from Fraunhover Portugal [12] is an open project that intends to familiarize elder people and people in general within their early stages of dementia. This system presents the positioning to the user through an arrow that rotates like a compass indicating the travel path. AlzNav also provides a localization system that allows the caregiver to send a Short Message Service (SMS) and receive the location of the person with needs of care.

\section{System Description}

One of the simplest ways to establish communication between caregivers and people with cognitive disabilities is through written messages. As an alternative, cellphones may be used and caregivers act as reminders to people with cognitive disabilities. Nowadays, cellphones are being replaced by smartphones. These devices may have 
several applications installed that help the user in almost every task of his/her life. However, there is a flaw when referring to applications specifically developed for people with disabilities.

This work describes a system that is being developed for this group of people. It is a localization system that helps not only the patient but also his/her caregiver. The main goal is to provide an application easy-to-use that helps the user that moves from one location to another. The localization capability lets the caregiver be aware of the user's current location. This feature may allow the caregiver to develop another activity without neglecting the care provided to the user.

The framework of this system (Figure 1) is divided into three major parts according to its destinated user: the application for the person with cognitive disabilities (designated by Cognitive Helper Mobile Solution, Section 3.1), two applications for his/her caregiver (Caregiver Applications - Mobile and Web, Section 3.2) and the server.

The server is composed by two modules: database and Communication Software. The database stores all the important data that is necessary for the correct operation of the system (like usernames and locations). The Communication Software ensures the communications established between the applications and the server.

\subsection{Cognitive Helper Mobile Application}

The person with cognitive disabilities has access to a mobile application that runs on Android Operative System, aiming at two main objectives: route the user so he/she may end at the pretended target (without getting lost) and locate the user, so caregivers may be aware of his/her actual location.

The detailed framework of this application is present in Figure 2 and it is divided into three parts. The localization of the user is retrieved through a smartphone's GPS module and by an Inertial Navigation System (INS) presented in [13]. The system, once it get the user location, may start the routing since it is possible to calculate the journey between the present location and the destination point.

To turn easier the user routing it is used augmented reality (Figure 3). To create this specific environment it is necessary to use the image captured from the smartphone's camera and using the sensors of the device (accelerometer and gyroscope); it is therefore possible to figure the direction the user is pointing the device. These

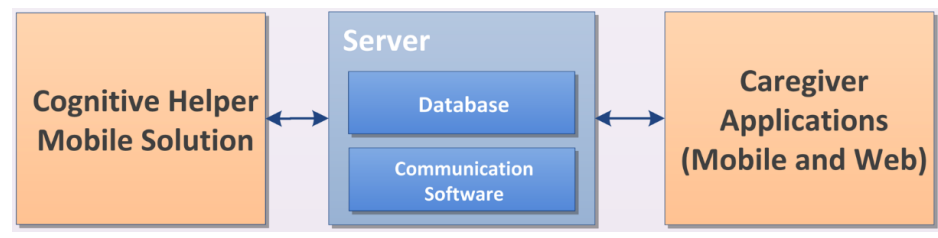

Fig. 1 Simplified framework of the system 


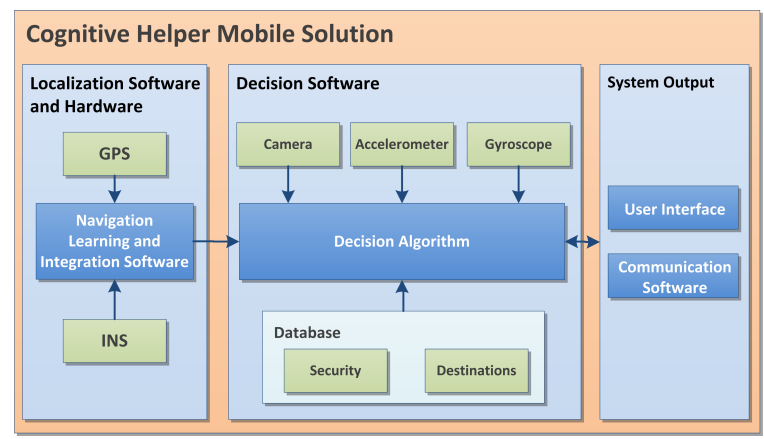

Fig. 2 Detailed framework of the mobile application for people with cognitive disabilities

three elements enable the augmented reality environment, letting the application know to where the user is turned to. A simple green arrow shows the travel path and the distance to be traveled to the next turn. With the localization and augmented reality is then possible to orientate the user and ensure that he/she is moving correctly. The Decision Algorithm (Figure 2) is responsible for this last task, creating alerts whenever necessary. If the user is at an intersection and is confused on where to go then he/she has to horizontally rotate the smartphone from one side to another and the green flag appears when the smartphone is pointing to the right path.

To interact with the user it is required to use an interface. This module shows, in an interactive way, the information to the user. The selection of a destination is executed through few menus and options. According to the type of destinations stored on the database the user may choose a fixed destination (more common location, like work place or home) or a normal one (generic location, like the mall). To keep this information confidential it is necessary to securely store this information in the database and guarantee that only the user has access to it.

The Communication Software establishes the connections between the application and the server. Through this module the application may update the user destinations, update his/her position or receive/send messages from/to his/her caregiver.

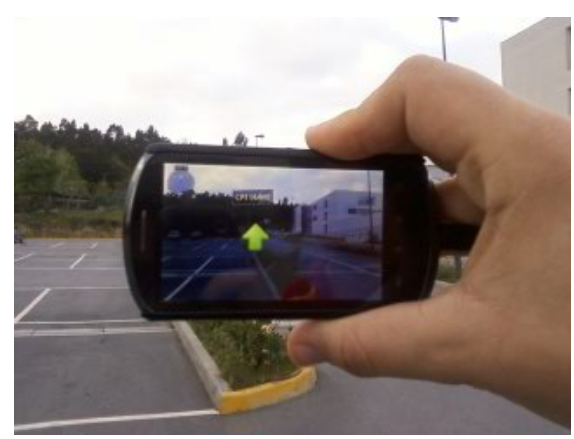

Fig. 3 Orientation system using augmented reality 
This application intends to create a navigation system that is easy-to-use, especially by people that may not be able to perform a complex mental task. When the caregiver believes the person with cognitive disabilities is capable to correctly use the application, it is possible to allow him/her to manually insert the destination address and not be limited to those that were previously created by the caregiver.

\subsection{Caregiver Applications}

Caregivers have two different applications with similar functions: a mobile application for Android OS and a Web application. The main goal of both applications is to let the caregiver be aware of the current location of the person with cognitive disabilities; it also presents all the walking paths accomplished from the starting point. The Web application has more capabilities since it is through it that a caregiver registers himself/herself on the platform and creates/adds usernames for the person with cognitive disabilities by whom is responsible.

The framework for these applications (Figure 4) is divided into four modules. The Communication Software ensures the transmission of the information between the applications and the server. This data includes not only caregiver's personal information (like his/her username, password, name) but also important data of the patient that he/she is responsible for (like location points, name, destinations stored on the database).

The caregiver is also in charge of the creation of the destination points (through Travel Path Designer) that may be used by the patient. This creation may be done by two different methods, i.e., either directly by selecting a point on a map or by searching a location through its address and, if necessary, adjusting the point on the map. Besides the creation of destinations it is the caregiver that specifies if they are starred (which is considered favorite and will be more used) or common ones.

Notifications Creator enables the caregiver to send simple messages of Yes or No type. This feature allows an easier and fast communication between caregivers and the people with cognitive disabilities. This feature may be useful when the caregiver is watching the traveling path, when it sees, for example, that the patient is passing by a grocery and asks if he/she can buy fruit. Instead of calling and pausing the

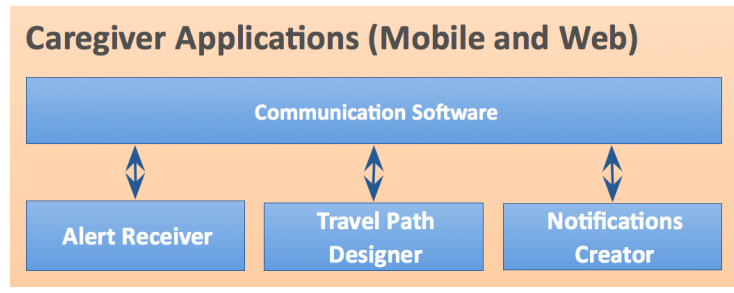

Fig. 4 Detailed framework of the applications for caregiver 
a)

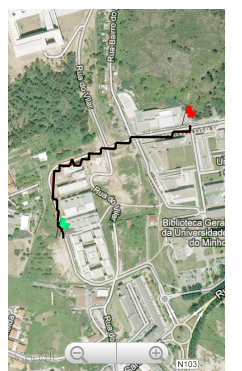

b)

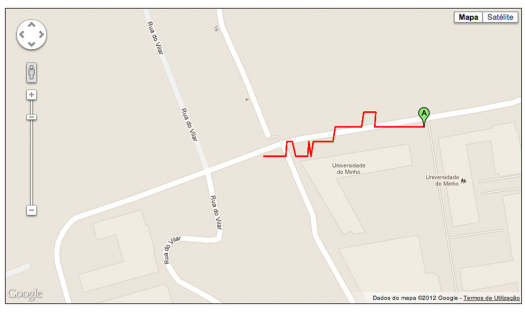

Fig. 5 Location system - a) Android application; b) Web application

navigation, this feature intends to enable a fast answer without interfering with the person routing.

The last module, Alert Receiver, receives all the alerts generated by Cognitive Helper Mobile Solution. This module informs the caregiver about any events created by the person with the cognitive disabilities, e.g., if the patient successfully reached his/her destination.

Figure 5a) represents the mobile application and shows a travel path that was already done by the person with cognitive disabilities. Figure 5b) represents the Web application and shows a travel path that is being done (the line is updated when the person with cognitive disabilities changes position). To indicate starting and ending points there is a marker on each one.

\section{Conclusion and Future Work}

Medicine allied to technology is being improved in the last years, but there are some diseases that still do not have a cure. Cognitive disabilities are an example of this situation and physicians tries to reduce the disease progression through medicines.

When it is diagnosed, the patient usually suffers from a reduction of his/her independence. This person is then not allowed to go outside his/her home alone and having a normal live. One of the major problems to people with cognitive disabilities is the lack of orientation, so the risk of getting lost once they go outside alone is very high.

This problem is being studied by several researchers that have been developing some applications/prototypes that route the user when he/she is outdoors.

The lack of orientation of these patients is then having a solution but caregivers still have a problem: when they are not present how do they know where the person with cognitive disabilities is? To answer this question the proposed system is being improved. Besides being an orientation system it is also a localization system.

The use of augmented reality in the orientation system turns it easier to use and surpass the limitations of similar systems, since it does not need static pictures that 
must be in the same landscape as that of the user, otherwise the way to understand the situation may be very complex.

The system is currently in a development stage and it is expected to do field tests in a near future with users, adding more features (e.g. detect preferred routes).

Acknowledgements This work is co-funded by National Funds through the FCT - Fundação para a Ciência e a Tecnologia (Portuguese Foundation for Science and Technology) within project PEst-OE/EEI/UI0752/2011. This work is part-funded by ERDF - European Regional Development Funds through the COMPETE Programme (operational programme for competitiveness) and by National Funds through the FCT - Fundao para a Ciłncia e a Tecnologia (Portuguese Foundation for Science and Technology) within project FCOMP-01-0124-FEDER-028980 (PTDC/EEI-SII/1386/2012). The work of João Ramos is also supported by a doctoral grant by FCT (SFRH/BD/89530/2012).

\section{References}

1. United Nations, Department of Economic and Social Affairs, Population Division: World Population Prospects: The 2010 Revision, New York (2011)

2. Sadri, F.: Multi-Agent Ambient Intelligence for Elderly Care and Assistance. In: Aip Conference Proceedings. Volume 2007., Aip (2007) 117-120

3. Augusto, J., Mccullagh, P.: Ambient Intelligence: Concepts and applications. Computer Science and Information Systems 4 (2007) 1-27

4. Stefanov, D.H., Bien, Z., Bang, W.C.: The smart house for older persons and persons with physical disabilities: structure, technology arrangements, and perspectives. IEEE Transactions on Neural and Rehabilitation Systems Engineering 12 (2004) 228-250

5. Carneiro, D., Novais, P., Costa, R., Gomes, P., Neves, J., Tscheligi, M., De Ruyter, B., Markopoulus, P., Wichert, R., Mirlacher, T., Meschterjakov, A., Reitberger, W.: EMon: Embodied Monitorization. Proceedings of the European Conference on Ambient Intelligence $\mathbf{5 8 5 9}(2009)$ 133-142

6. Alper, S., Raharinirina, S.: Assisitive Technology for Individuals with Disabilities: A Review and Synthesis of the Literature. Journal of Special Education Technology 21 (2006) 47-64

7. Dawe, M.: "Let Me Show You What I Want": Engaging Individuals with Cognitive Disabilities and their Families in Design. Technology (2007) 2177-2182

8. Dawe, M.: Desperately seeking simplicity: how young adults with cognitive disabilities and their families adopt assistive technologies. In: Proceedings of the SIGCHI conference on Human Factors in computing systems. CHI '06, ACM (2006) 1143-1152

9. Ramos, J., Anacleto, R., Costa, Â., Novais, P., Figueiredo, L., Almeida, A.: Orientation System for People with Cognitive Disabilities. In: Ambient Intelligence Software and Applications. Volume 153 of Advances in Intelligent and Soft Computing., Springer Berlin / Heidelberg (2012) 43-50

10. Carmien, S., Dawe, M., Fischer, G., Gorman, A., Kintsch, A., Sullivan, J.F.: Socio-technical environments supporting people with cognitive disabilities using public transportation. ACM Transactions on Computer-Human Interaction 12 (2005) 233-262

11. Liu, A.L., Hile, H., Borriello, G., Kautz, H., Brown, P.A., Harniss, M., Johnson, K.: Informing the Design of an Automated Wayfinding System for Individuals with Cognitive Impairments. Cognition 9 (2009) 1-8

12. Fraunnhover Portugal: AlzNav (2012)

13. Anacleto, R., Figueiredo, L., Novais, P., Almeida, A.: Providing location everywhere. In: Progress in Artificial Intelligence - Proceedings of the 15th Portuguese conference on Artificial Intelli- gence - EPIA 2011. (2011) 15-28 